\title{
Streptococcus suis Serotype 2 Capsule In Vivo
}

\section{Jean-Philippe Auger, ${ }^{1}$ Nattakan Meekhanon, ${ }^{1}$ Masatoshi Okura, ${ }^{1}$ Makoto Osaki, Marcelo Gottschalk, Tsutomu Sekizaki, Daisuke Takamatsu}

Many Streptococcus suis isolates from porcine endocarditis in slaughterhouses have lost their capsule and are considered avirulent. However, we retrieved capsule- and virulence-recovered $S$. suis after in vivo passages of a nonencapsulated strain in mice, suggesting that nonencapsulated S. suis are still potentially hazardous for persons in the swine industry.

Streptococcus suis is a gram-positive bacterium that $\mathcal{N}$ infects pigs and causes severe economic losses to the swine industry. Moreover, it causes severe disease in persons in close contact with diseased pigs or their products (1). In Japan, S. suis has been frequently isolated from pigs with endocarditis in slaughterhouses; most of the isolates were expected to be sequence types (STs) that are potentially hazardous to humans (2). Many isolates from porcine endocarditis lost their capsule, and all the nonencapsulated isolates analyzed had mutations in the capsular polysaccharide synthesis (cps) genes $(3,4)$. The capsule of $S$. suis is a major virulence factor (1). Although loss of the capsule gives $S$. suis some benefit in causing endocarditis by enhancing the ability of bacterial cells to adhere to porcine and human platelets, a major virulence determinant for infective endocarditis (3), nonencapsulated S. suis are generally considered avirulent (5). However, whether nonencapsulated $S$. suis lurking in porcine endocarditis poses a threat to persons working in the swine industry is unknown. To investigate whether nonencapsulated $S$. suis can restore the ability to express the capsule and become virulent again, we repeated in vitro or in vivo passages of nonencapsulated S. suis and attempted to retrieve capsule-recovered strains.

\section{The Study}

For the in vitro passages, we used 29 S. suis strains isolated from pigs with endocarditis. These isolates had the cps gene cluster of serotype 2 but had lost their capsule

Author affiliations: Université de Montréal, St-Hyacinthe, Quebec, Canada (J.-P. Auger, M. Gottschalk); The University of Tokyo, Tokyo, Japan (N. Meekhanon, T. Sekizaki); Kasetsart University, Bangkok, Thailand (N. Meekhanon); National Agriculture and Food Research Organization, Tsukuba, Japan (M. Okura, M. Osaki, D. Takamatsu); Gifu University, Gifu, Japan (D. Takamatsu)

DOI: http://dx.doi.org/10.3201/eid2210.151640 because of mutations in the cps genes (Table). We subcultured them twice in liquid media and separated the cells according to the buoyant density by Percoll density gradient centrifugation (online Technical Appendix, http://wwwnc. cdc.gov/EID/article/22/10/15-1640-Techapp1.pdf). Because encapsulated cells show lower density than nonencapsulated cells $(6,7)$, we investigated capsular expression of $S$. suis cells with low density by coagglutination tests using serotype 2 antiserum (online Technical Appendix). The retrieved S. suis was also used for the next subcultures. We repeated 4 cycles of this experiment (in total 8 subcultures) but obtained no encapsulated $S$. suis from any of the strains tested.

Although these results suggested that mutations in cps genes are not repaired easily, the conditions faced by $S$. suis in vivo could influence capsular expression. To investigate this possibility, we selected strain NL119 as a representative. NL119 is an ST1 strain, one of the types hazardous to humans, but one that has lost the capsule because of a point mutation that occurred at nt 490 (T490C, Cys164Arg) of a glycosyltransferase gene (cps2F) (Table; Figure 1, panel A) (4). We inoculated groups of 5 mice with $5 \times 10^{8} \mathrm{CFU}$ of NL119 (online Technical Appendix). Bacteria persistent in mice were retrieved $36 \mathrm{~h}$ after infection from the blood, in which capsular expression works favorably for survival. We investigated capsular expression of the retrieved NL119 by coagglutination tests and used the colony giving the strongest reaction within $30 \mathrm{~s}$ for the subsequent in vivo passage.

As expected, the coagglutination test of the parental strain NL119 showed a negative result. Similarly, NL119 after the first and second passages (NL119 P1 and P2, respectively) reacted weakly, comparable to those of the parental strain, suggesting poor encapsulation. Meanwhile, NL119 after the third and fourth passages (NL119 P3 and $\mathrm{P} 4$, respectively) reacted strongly, suggesting recovery of the capsule. To confirm this finding, we further analyzed formalin-killed bacteria by dot-ELISA using monoclonal antibody Z3, which reacts with the sialic acid moiety of the serotype 2 capsule (8), and an anti-S. suis serotype 2 serum adsorbed with parental strain NL119 to select the capsule-specific antibodies (online Technical Appendix). In accordance with the coagglutination test, NL119 P1 and P2 gave weak reactions similar to those of NL119, whereas strong signals were detected in NL119 P3 and P4 with both the monoclonal antibody and serum (Figure 1, panels B, C). Because NL119 P1-P4 were also ST1 as determined by multilocus sequence

${ }^{1}$ These authors contributed equally to this article. 
Table. Nonencapsulated Streptococcus suis strains isolated from pigs with endocarditis and used for in vitro passages to investigate possible capsule recovery

\begin{tabular}{|c|c|c|c|c|}
\hline Strain & Affected gene(s) & Types of mutations & Affected nucleotide(s) (affected amino acid) & Reference \\
\hline NL100 & $c p s 2 F$ & Nonsense & T696G (Tyr232TERM) & $(4)$ \\
\hline NL119 & $c p s 2 F$ & Missense & T490C (Cys164Arg) & (4) \\
\hline NL122 & $c p s 2 F$ & Missense & G52A (Gly18Ser) & (4) \\
\hline NL126 & $c p s 2 F$ & Frameshift by insertion & TCCG & (4) \\
\hline \multirow[t]{2}{*}{ NL132 } & $c p s 2 E$ & Missense & G1199A (Arg400Lys) & (4) \\
\hline & $c p s 2 H$ & Frameshift by deletion & TA & (4) \\
\hline \multirow[t]{3}{*}{ NL143 } & $c p s 2 F$ & Missense & G493T (Asp165Tyr) & (4) \\
\hline & $\operatorname{cps} 2 K$ & Insertion & AATCATTGG & (4) \\
\hline & cps $2 R$ & Missense & G496A (Gly166Arg) & (4) \\
\hline NL146 & $c p s 2 F$ & Nonsense & T482A (Leu162TERM) & (4) \\
\hline NL171 & $\operatorname{cps} 2 E$ & Insertion & IS element: 1,619 bp & (3) \\
\hline NL174 & cps2H & Frameshift by deletion & A & (4) \\
\hline NL175 & $c p s 2 H$ & Frameshift by deletion & A & (4) \\
\hline NL184 & $c p s 2 E$ & Insertion & IS element: $1,115 \mathrm{bp}$ & (3) \\
\hline NL194 & $c p s 2 E$ & Insertion & IS element: 1,416 bp & (3) \\
\hline NL208 & $c p s 2 E$ & Frameshift by deletion & TAAG & (4) \\
\hline NL219 & $c p s 2 E$ & Frameshift by deletion & TAAG & (4) \\
\hline NL225 & $c p s 2 F$ & Frameshift by insertion & CCAAA & (4) \\
\hline NL230 & $c p s 2 F$ & Frameshift by insertion & A & (4) \\
\hline NL240 & $c p s 2 E$ & Nonsense & C1189T (GIn397TERM) & (4) \\
\hline NL245 & $c p s 2 E$ & Frameshift by insertion & $\mathrm{T}$ & (4) \\
\hline NL249 & $c p s 2 E$ & Frameshift by insertion & AGCA & (4) \\
\hline NL255 & $c p s 2 E$ & Insertion & IS element: 1,619 bp & (3) \\
\hline NL257 & $c p s 2 E$ & Frameshift by insertion & ATCT & (4) \\
\hline NL266 & $c p s 2 E$ & Frameshift by deletion & A & (4) \\
\hline NL278 & $c p s 2 F$ & Missense & T259C (Ser87Pro) & (4) \\
\hline NL295 & $c p s 2 F$ & Missense & T492G (Cys164Trp) & (4) \\
\hline NL303 & $c p s 2 F$ & Deletion & $81 \mathrm{bp}$ & (4) \\
\hline \multirow[t]{2}{*}{ NL322 } & cps2B & Missense & G469A (Asp157Asn) & (4) \\
\hline & $c p s 2 G$ & Deletion & $50 \mathrm{bp}$ & (4) \\
\hline NL328 & $c p s 2 F$ & Frameshift by deletion & $A G$ & (4) \\
\hline NL342 & $c p s 2 E$ & Frameshift by deletion & TAAG & (4) \\
\hline \multirow[t]{2}{*}{ NL345 } & $c p s 2 \mathrm{H}$ & Deletion & $23 \mathrm{bp}$ & (4) \\
\hline & cps $2 N$ & Missense & C706T (Pro236Ser) & (4) \\
\hline
\end{tabular}

typing, these results suggested that NL119 had recovered the capsule during passages in animals.

To find mutations that had contributed to the capsule recovery, we sequenced the $c p s 2 F$ gene of NL119 P1-P4. Although the cytosine residue at nt 490 was not changed in comparison with the parental strain, we found a further missense mutation at nt 491 (G491C, Arg164Pro) of the cps $2 F$ gene in NL119 P3 and P4 (Figure 1, panel A). To investigate whether this mutation was involved in the capsule recovery, we cloned $\operatorname{cps} 2 F$ of NL119 P4 into a gene expression vector pMX1 (9) and introduced it into the parental strain NL119 (online Technical Appendix). A coagglutination test using serotype 2 antiserum showed positive reactions in all transformants tested, demonstrating that the further missense mutation restored the function of $\operatorname{cps} 2 F$, resulting in capsule recovery of NL119 P3 and P4, although how the $\mathrm{Cps} 2 \mathrm{~F}$ function was recovered by the amino acid substitution is unknown. Isolation of the capsule-recovered strains in vivo could have been the consequence of selection of encapsulated cells, which were already present as a subpopulation in the original nonencapsulated NL119 population, by resisting host immunity including phagocytosis. However, because NL119 was a nonencapsulated strain originally recovered from a single colony and well-isolated by repeated passages in vitro, and no encapsulated subpopulation was ever retrieved in vitro by the selection using Percoll density gradient centrifugation, the most plausible hypothesis would be that the capsule-recovered $S$. suis was generated in vivo.

To evaluate whether the capsule-recovered S. suis isolate also recovered its virulence, we infected mice with either NL119 or NL119 P4 (online Technical Appendix). Rates of death differed significantly $(\mathrm{p}<0.05)$ : $50 \%$ death in the NL119 P4-infected mice 14 days after infection, compared with $0 \%$ for the nonencapsulated NL119 (Figure 2 , panel A). Recovery of the capsule also significantly increased its survival in blood $24 \mathrm{~h}$ after infection $(\mathrm{p}<0.05)$. All but 1 surviving NL119 P4-infected mice had significant blood bacterial titers $\left(\geq 5 \times 10^{3} \mathrm{CFU} / \mathrm{mL}\right.$; geometric mean $10^{4} \mathrm{CFU} / \mathrm{mL}$ ). In contrast, except for 1 mouse, all mice infected with NL119 had blood bacterial titers $<10^{4} \mathrm{CFU} / \mathrm{mL}$ (geometric mean $10^{2} \mathrm{CFU} / \mathrm{mL}$ ) (Figure 2, panel B).

\section{Conclusions}

Although capsule loss might contribute to $S$. suis infection by enhancing bacterial adherence to host cells and 
Figure 1. Capsule recovery of Streptococcus suis strain NL119 in vivo. A) The genetic organization of the S. suis serotype 2 capsular polysaccharide synthesis (cps) gene cluster and mutations observed in isolate NL119 and strains retrieved from NL119infected mice after each in vivo passage (NL119 P1-P4; DDBJ/ EMBL/GenBank accession nos. LC147077, LC147078, LC147079, LC147080, and LC077855, respectively). Gray arrows indicate genes putatively involved in capsule synthesis; open arrows indicate genes with unknown functions; numbers indicate nucleotide positions in cps2F. NL119 lost the ability to synthesize the capsule because of a missense mutation at nt 490 (T490C, Cys164Arg) of cps2F

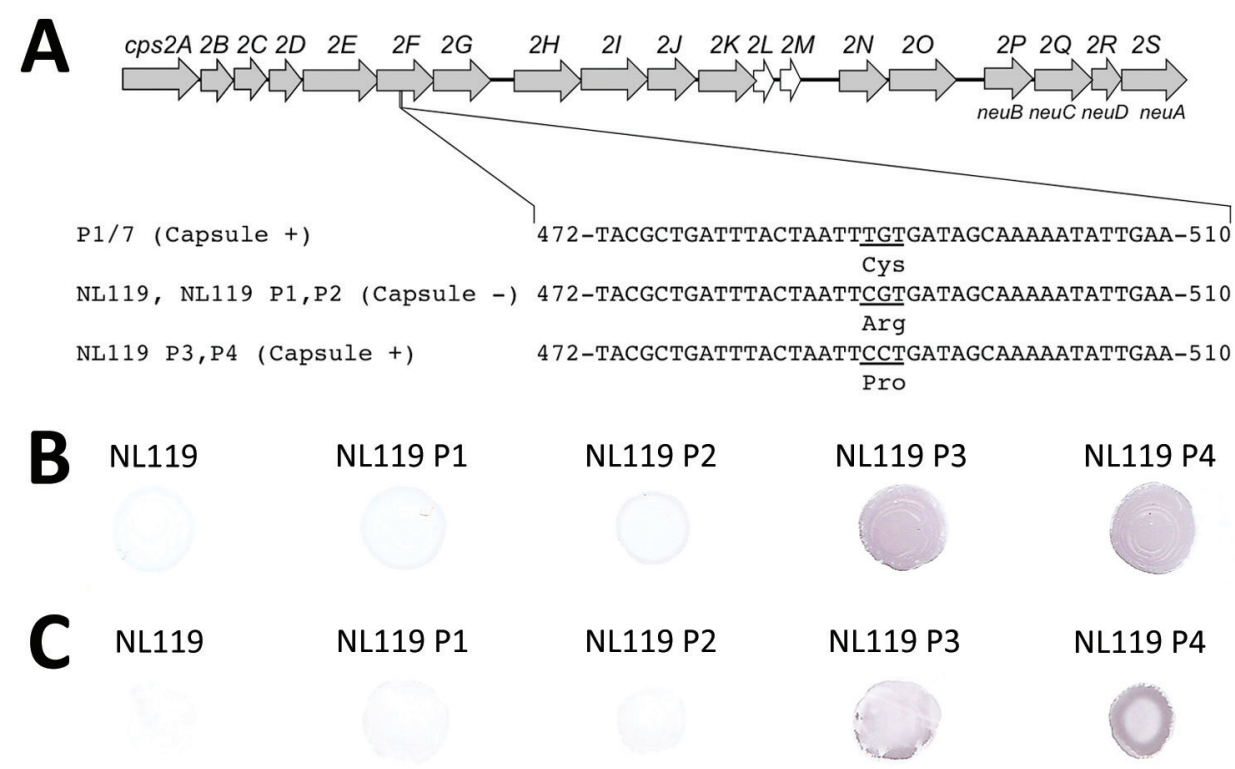

(4). B, C) NL119 P1 and P2 retrieved from mice after the first and second in vivo passages, remained nonencapsulated, and their cps2F sequences were identical to that of NL119. In NL119 P3 and P4 retrieved after the third and fourth passages a further missense mutation at $\mathrm{nt} 491$ (G491C, Arg164Pro) of $c p s 2 F$ restored the function of the gene, resulting in capsule recovery of the strains. Dot-ELISA of NL119 and strains retrieved from NL119-infected mice after each in vivo passage (NL119 P1-P4) using monoclonal antibody Z3 (B) and polyclonal anti-S. suis serotype 2 serum adsorbed with NL119 (C). Monoclonal antibody Z3 specifically recognizes the sialic acid moiety of the $S$. suis serotype 2 capsule.

biofilm formation $(3,10-12)$, capsule loss makes S. suis cells susceptible to phagocytosis; therefore, the virulence of nonencapsulated mutants was attenuated when evaluated in animal models (5). In accordance with previous studies, nonencapsulated NL119 was avirulent. However, NL119 P4, which recovered its capsule in vivo, also recovered virulence. Because various mutations in cps genes, including large deletions and insertions, cause capsule loss in $S$. suis $(3,4)$, not all mutations will be repaired like NL119. However, our results demonstrated the presence of a nonencapsulated mutant, which can recover the capsule and virulence in vivo. Hence, nonencapsulated $S$. suis strains can cause severe diseases to the next hosts by recovering the capsule, which indicates that some nonencapsulated $S$. suis lurking in pigs with endocarditis are still potentially hazardous to
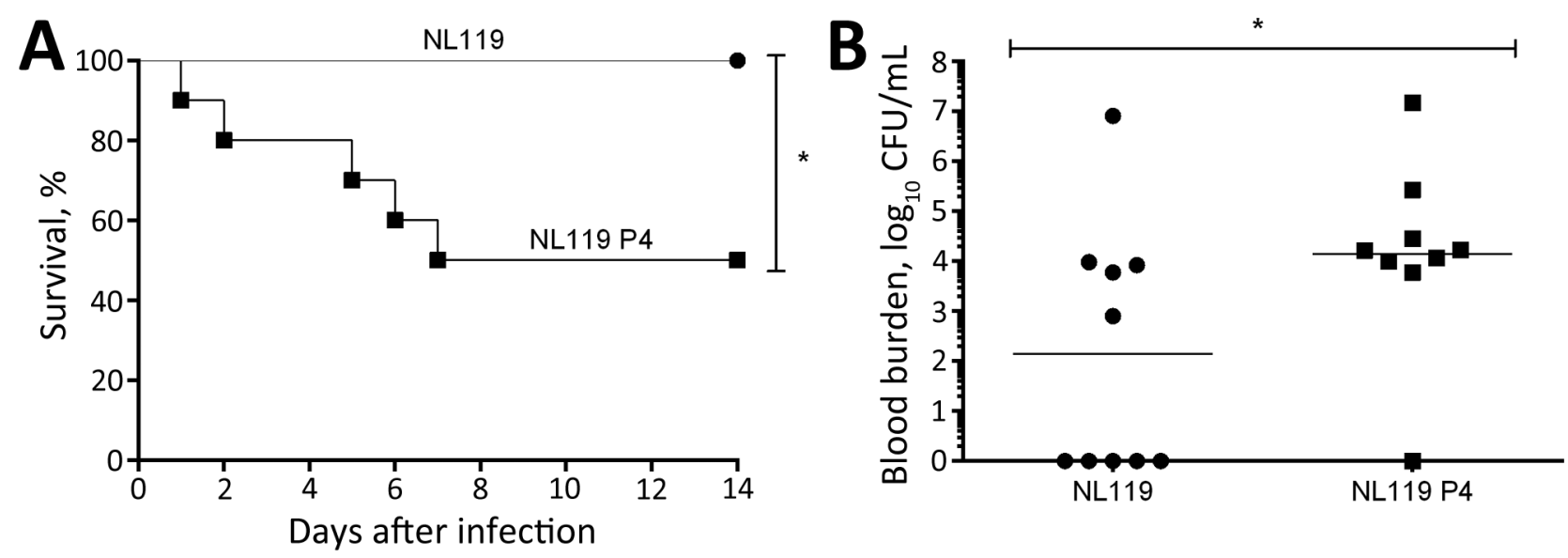

Figure 2. Virulence of nonencapsulated Streptococcus suis strain NL119 and capsule-recovered NL119 P4 in mice. A) Survival of C57BL/6 mice ( $n=10$ mice per strain; until 14 days after infection) inoculated intraperitoneally with $5 \times 10^{7} \mathrm{CFU}$ of either NL119 or NL119 P4. B) Blood bacterial burden at $24 \mathrm{~h}$ after infection. Data of individual mice are presented as $\log _{10} \mathrm{CFU} / \mathrm{mL}$ with the geometric mean. Asterisks indicate a significant difference between NL119 and NL119 P4 $(p<0.05)$. 
persons handling such pigs and their products. Further investigations using a variety of naturally occurring and laboratory-derived mutants are needed for a comprehensive understanding of the biological significance and mechanisms of this phenomenon.

\section{Acknowledgments}

We thank Sonia Lacouture for excellent technical assistance.

This work was financially supported by Japan Society for the Promotion of Science (JSPS) KAKENHI grant no. 26870840 to M.O.; JSPS KAKENHI grant nos. 23580420, 26660226, and $15 \mathrm{H} 02651$ to T.S.; and Natural Science and Engineering Research Council of Canada (NSERC) 04435 to M.G.

Mr. Auger is a doctoral student at the Research Group on Infectious Diseases of Swine at the Université de Montréal in St-Hyacinthe, Quebec, Canada. His primary research interest is the host response to pathogens.

\section{References}

1. Gottschalk M. Streptococcosis. In: Zimmerman JJ, Karriker LA, Ramirez A, Schwartz KJ, Stevenson GW, editors. Diseases of swine, 10th ed. Ames (IA): John Wiley \& Sons, Inc.; 2012. p. 841-55.

2. Onishi H, Sugawara M, Okura M, Osaki M, Takamatsu D. Prevalence of Streptococcus suis genotypes in isolates from porcine endocarditis in East Japan. J Vet Med Sci. 2012;74:1681-4. http://dx.doi.org/10.1292/jvms.12-0301

3. Lakkitjaroen N, Takamatsu D, Okura M, Sato M, Osaki M, Sekizaki T. Loss of capsule among Streptococcus suis isolates from porcine endocarditis and its biological significance. J Med Microbiol. 2011;60:1669-76. http://dx.doi.org/10.1099/jmm.0.034686-0

4. Lakkitjaroen N, Takamatsu D, Okura M, Sato M, Osaki M, Sekizaki T. Capsule loss or death: the position of mutations among capsule genes sways the destiny of Streptococcus suis. FEMS Microbiol Lett. 2014;354:46-54. http://dx.doi.org/10.1111/15746968.12428
5. Fittipaldi N, Segura M, Grenier D, Gottschalk M. Virulence factors involved in the pathogenesis of the infection caused by the swine pathogen and zoonotic agent Streptococcus suis. Future Microbiol. 2012;7:259-79. http://dx.doi.org/10.2217/fmb.11.149

6. Håkansson S, Bergholm A-M, Holm SE, Wagner B, Wagner M. Properties of high and low density subpopulations of group B streptococci: enhanced virulence of the low density variant. Microb Pathog. 1988;5:345-55. http://dx.doi.org/10.1016/08824010(88)90035-6

7. Patrick S, Reid JH. Separation of capsulate and non-capsulate Bacteroides fragilis on a discontinuous density gradient. J Med Microbiol. 1983;16:239-41. http://dx.doi.org/10.1099/0022261516-2-239

8. Charland N, Jacques M, Lacouture S, Gottschalk M. Characterization and protective activity of a monoclonal antibody against a capsular epitope shared by Streptococcus suis serotypes 1, 2 and 1/2. Microbiology. 1997;143:3607-14. http://dx.doi.org/10.1099/00221287-143-11-3607

9. Okura M, Osaki M, Fittipaldi N, Gottschalk M, Sekizaki T, Takamatsu D. The minor pilin subunit Sgp2 is necessary for assembly of the pilus encoded by the $\operatorname{srt} G$ cluster of Streptococcus suis. J Bacteriol. 2011;193:822-31. http://dx.doi.org/10.1128/JB.01555-09

10. Benga L, Goethe R, Rohde M, Valentin-Weigand P. Non-encapsulated strains reveal novel insights in invasion and survival of Streptococcus suis in epithelial cells. Cell Microbiol. 2004; 6:867-81. http://dx.doi.org/10.1111/j.1462-5822.2004.00409.x

11. Bonifait L, Gottschalk M, Grenier D. Cell surface characteristics of nontypeable isolates of Streptococcus suis. FEMS Microbiol Lett. 2010;311:160-6. http://dx.doi.org/10.1111/ j.1574-6968.2010.02086.x

12. Tanabe S, Bonifait L, Fittipaldi N, Grignon L, Gottschalk M, Grenier D. Pleiotropic effects of polysaccharide capsule loss on selected biological properties of Streptococcus suis. Can J Vet Res. 2010;74:65-70.

Address for correspondence: Daisuke Takamatsu, Division of Bacterial and Parasitic Diseases, National Institute of Animal Health, National Agriculture and Food Research Organization, 3-1-5 Kannondai, Tsukuba, Ibaraki 305-0856, Japan; email: p1013dt@affrc.go.jp

\section{EID Podcast: Shigella Sonnei and Shiga Toxin}

Shiga toxins (Stx) are primarily associated with Shiga toxinproducing Escherichia coli and Shigella dysenteriae serotype 1. Stx production by other shigellae is uncommon, but in 2014, Stx1-producing S. sonnei infections were detected in California. During June 2014-April 2015, 56 cases of Stx1-producing $S$. sonnei were identified, in 2 clusters. Continued surveillance of Stx1-producing S. sonnei in California is necessary to characterize its features and plan for reduction of its spread in the United States.

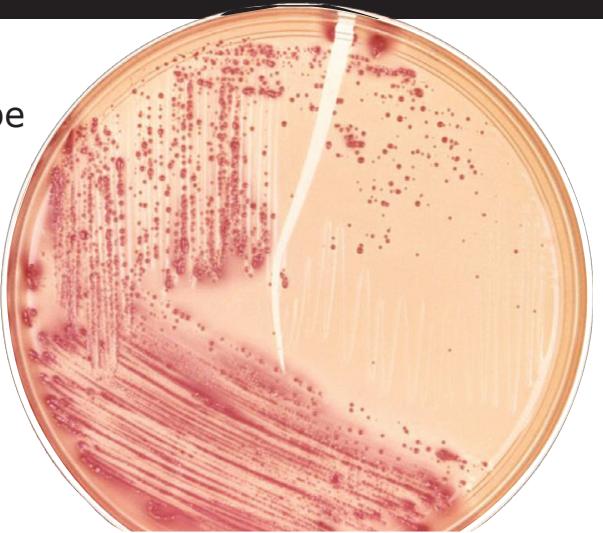

